\title{
Impact of pilot sequence contamination in massive MIMO systems
}

DOI:

10.1049/iet-com.2017.0062

\section{Document Version}

Accepted author manuscript

Link to publication record in Manchester Research Explorer

\section{Citation for published version (APA):}

AlKhaled, M., \& Alsusa, E. (2017). Impact of pilot sequence contamination in massive MIMO systems. IET Communications, 11(13), 2005-2011. https://doi.org/10.1049/iet-com.2017.0062

\section{Published in:}

IET Communications

\section{Citing this paper}

Please note that where the full-text provided on Manchester Research Explorer is the Author Accepted Manuscript or Proof version this may differ from the final Published version. If citing, it is advised that you check and use the publisher's definitive version.

\section{General rights}

Copyright and moral rights for the publications made accessible in the Research Explorer are retained by the authors and/or other copyright owners and it is a condition of accessing publications that users recognise and abide by the legal requirements associated with these rights.

\section{Takedown policy}

If you believe that this document breaches copyright please refer to the University of Manchester's Takedown Procedures [http://man.ac.uk/04Y6Bo] or contact uml.scholarlycommunications@manchester.ac.uk providing relevant details, so we can investigate your claim.

\section{OPEN ACCESS}




\section{On the Impact of Pilot Sequence Contamination in Massive MIMO Systems}

\section{Makram AlKhaled ${ }^{1}$ and Emad Alsusa ${ }^{2}$}

School of Electrical and Electronic Engineering, The University of Manchester, United Kingdom, M13 9PL.

${ }^{1}$ Makram.Alkhaled@manchester.ac.uk, ${ }^{2}$ E.Alsusa@manchester.ac.uk

Abstract: Time-division duplex (TDD) is the most efficient technique for acquiring channel state information (CSI) in massive MIMO systems where the reciprocity between the uplink and downlink channels is utilised by the pilot signals to extract the channel parameters. In this paper, we consider the pilot contamination problem in TDD multicell multiuser massive MIMO systems and examine two different pilot signal allocation schemes for which we derive the lower bounds on the achievable rate on the uplink for the cases of maximum-ratio combining (MRC) and zero-forcing (ZF) detectors. To achieve further performance enhancements, we propose a new algorithm for pilot sequences allocation in which the multiplicity of the pilot sequences over the number of users in each cell is exploited. Our results show that when pilot contamination is severe, allocating more system resources for channel estimation results in a better system performance especially in limited mobility environments. Moreover, we show that when the signal to interference plus noise ratio (SINR) is low, MRC is superior to ZF, and vice versa. Finally, we demonstrate that our proposed allocation algorithm can significantly improve the spectral efficiency of the network compared to the conventional pilot allocation method.

\section{Introduction}

Multiuser multiple-input multiple-output (MU-MIMO) systems are likely to be an integral part of future wireless communication systems due to their ability to provide high data rates and reliability [1]. While most modern cellular standards, such as 802.16 (WiMAX) and LTE-Advanced, employ several antennas per base station (BS), the improvement in the data rate is relatively modest. Recently, massive MIMO systems have been proposed to reap more of the benefits of conventional MU-MIMO systems by increasing the number of BS antennas on a much larger scale [2]. The basic idea of massive MIMO systems is to use a large number of antennas (100 or more) at the BS to provide services to a few number of users in the same time-frequency resources [3]. It has been 
practically and theoretically proven that massive MIMO systems can significantly enhance both energy and spectral efficiencies [4][5]. These enhancements however can be efficiently achieved with time-division duplex (TDD) scheme, which does not require a dedicated feedback channel [6]. However, the pilot sequences are limited in number and length and therefore as the number of users increases, it becomes impossible for each user to have a unique pilot sequence. Hence, the same pilot sequences are reused in different cells which makes the pilots experience inter-cell interference. This problem is more commonly known in the literature as "pilot contamination" $[2]$.

Pilot contamination limits the performance of multicell massive MIMO systems in both downlink and uplink. Thus, several methods have been proposed to mitigate or reduce the effect of pilot contamination. In [7], a precoding method that can mitigate the pilot contamination using multicell cooperation was proposed. However, the information exchange overhead required between the cooperating BSs increases in proportion to the number of BSs antennas. Therefore, this precoding method is only suitable for MIMO systems with a small number of antennas. The authors in [8] have shown that users with mutually non-overlapping angle-of-arrival (AOA) hardly contaminate each other, even if they use the same pilot sequences. Based on this, a coordinated scheme for assigning pilot sequences only to users of this type was proposed. However, this method requires that the AOA of each user to be small. In [9], a greedy pilot allocation algorithm is proposed which employs the statistical channel covariance information to mitigate the effect of pilot contamination. However, this algorithm suffers from high complexity. To the best of the authors' knowledge, none of the published work in literature has considered allocating more system resources to mitigate the negative impact of pilot contamination, except [10]. In [10] the authors proposed a soft pilot reuse (SPR) scheme that uses larger number of pilot sequences to improve the quality of service for the edge users. The SPR scheme is based on comparing the channel quality of each user with a threshold value. However, finding the optimum threshold value significantly increases the complexity of the system.

In this paper, we consider two different scenarios for pilot sequence allocation. In the first 
scenario, we consider the case of no pilot contamination effect by assuming that none of the pilot sequences assigned to any user in the system is re-used by any other user, which means higher usage of system resources for channel estimation. While in the second scenario, we assume that the pilot sequences used in one cell are re-used by the users of the other cells, which means allocating more system resources for data transmission but with the existence of pilot contamination. To evaluate the system performance in each scenario, we derive lower bounds on the achievable rates for two linear detection techniques, namely maximum-ratio combining (MRC) and zero-forcing (ZF). Furthermore, we investigate the performance of the system with both scenarios under different system settings. Based on the results of these comparisons we conclude which scenario and which uplink detector is more suitable for each setting. Finally, we consider the case when there is a multiplicity of pilot sequences over the users in each cell, which is the case when either the number of users is not very large or by allocating more system resources for the channel estimation process. We propose a pilot allocation algorithm that utilizes this multiplicity to reduce the effect of pilot contamination and improve the system performance compared to the conventional pilot allocation method. Unlike [10], in our proposed algorithm we assume that the number and the length of the pilot sequences are predefined and represent a fixed percentage of the coherence time of the channel. This assumption is more realistic, and it reduces the complexity burden of the proposed algorithm compared to other benchmark algorithms.

The rest of the paper is organised as follows. The system model is described in Section II. In Section III we illustrate the communication scheme and we discuss the considered scenarios for pilot signals allocation. We analyse the system performance and derive lower bounds on the achievable rates in Section IV. The proposed pilot allocation algorithm is presented in Section V. Numerical results are presented and discussed in Section VI. Finally, conclusions are drawn in Section VII.

Notation: Bold font lower and upper case symbols represent vectors and matrices, respectively. The inverse operation and the Hermitian transpose operators are denoted by $(\cdot)^{-1}$ and $(\cdot)^{\dagger}$, respectively. $[\boldsymbol{A}]_{i j}$ represents the $(i, j)$ th entry of the matrix $\boldsymbol{A} . \boldsymbol{I}_{K}$ is the identity matrix of size 
$K \times K$. Finally, the norm of a vector and the expectation operation are denoted by $\|\cdot\|$ and $\mathbb{E}[\cdot]$, respectively.

\section{System Model}

We consider the uplink of a multicell massive MIMO system with $L$ cells numbered $1,2, \ldots, L$ which are sharing the same frequency band. Each cell is equipped with one BS that utilises $M$ service antennas to provide services to $K$ single antenna users. The users in each cell transmit data to their corresponding $\mathrm{BS}$ in the same time-frequency resource. Hence, the $M \times 1$ received signals vector at the BS of the $l$-th cell is given by

$$
\boldsymbol{y}_{l}=\sqrt{p_{u}} \sum_{i=1}^{L} \boldsymbol{G}_{l i} \boldsymbol{x}_{i}+\boldsymbol{n}_{l},
$$

where $p_{u}$ is the normalized transmit SNR, $\boldsymbol{x}_{i}$ is the $K \times 1$ vector of symbols transmitted simultaneously by the $K$ users in the $i$-th cell, $\boldsymbol{n}_{l}$ is the $M \times 1$ vector of additive white zero-mean unit-variance Gaussian noise, and $\boldsymbol{G}_{l i}$ is the $M \times K$ channel matrix between the $K$ users in the $i$-th cell and the $M$ antennas of the BS of the $l$-th cell. The channel matrix $\boldsymbol{G}_{l i}$ models the independent fast fading, the geometrical attenuation, and the log-normal shadowing and can be represented as

$$
\boldsymbol{G}_{l i}=\boldsymbol{H}_{l i} \boldsymbol{D}_{l i}^{1 / 2}
$$

where $\boldsymbol{H}_{l i}$ is the $M \times K$ matrix of fast fading coefficients between the $K$ users of the $i$-th cell and the $M$ antennas of the $l$-th BS, i.e., $\left[\boldsymbol{H}_{l i}\right]_{m k}=h_{\text {limk }}$ is the fast fading coefficient between the $k$-th user in the $i$-th cell to the $m$-th antenna in the $l$-th BS. The elements of $\boldsymbol{H}_{l i}$ matrix are assumed to be independent and identically distributed (i.i.d) zero-mean, circularly-symmetric complex Gaussian $\mathcal{C N}(0,1)$ random variables. The matrix $\boldsymbol{D}_{l i}$ is a diagonal matrix of size $K \times K$, where $\left[\boldsymbol{D}_{l i}\right]_{k k}=\beta_{l i k}$ represents the large scale fading coefficient between the $k$-th user in the $i$-th cell and the $l$-th BS. We assume that the large scale fading coefficients to be constant over many coherence intervals, 
independent over the antenna index $m$, and known to everybody.

\section{Communication Scheme}

The communication scheme for the system considered in this paper consists of the following two phases:

\subsection{Uplink Training and Channel Estimation}

We assume that an interval of length $\tau$ is used for uplink training, which is shorter than the coherence time of the channel $T$. We further assume that the users in all the cells simultaneously transmit pilot sequences of length $\tau$. Using the received pilot sequences, the BSs estimate the uplink CSI to build the detection matrix.

We consider two different scenarios for pilot sequences allocation, namely, orthogonal pilots and pilot re-use. To differentiate between the two cases, we use the subscripts $(\cdot)_{o}$ and $(\cdot)_{r}$ to refer to the first and the second scenario, respectively.

\subsubsection{Orthogonal pilots}

Here, we assume that different orthogonal pilot sequences are assigned to the users in the system, and none of these pilot sequences is re-used by any other user in any other cell. To ensure orthogonality in this case, the length of the pilot sequence $\tau_{o}$ should satisfy $\tau_{o} \geq L K$. Then, the $M \times \tau_{o}$ matrix of received pilot signals at the $l$-th BS can be represented as

$$
\boldsymbol{Y}_{l, o}=\sqrt{p_{o}} \sum_{i=1}^{L} \boldsymbol{G}_{l i} \boldsymbol{\Phi}_{i, o}+\boldsymbol{N}_{l, o}
$$

where $p_{o} \triangleq \tau_{o} p_{u}, N_{l, o}$ is the $M \times \tau_{o}$ AWGN matrix at the $l$-th BS with i.i.d $C \mathcal{N}(0,1)$ elements, and $\boldsymbol{\Phi}_{i, o}$ is the $K \times \tau_{o}$ matrix of pilot sequences transmitted by the $K$ users in the $i$-th cell which satisfies 


$$
\boldsymbol{\Phi}_{i, o} \boldsymbol{\Phi}_{l, o}^{\dagger}= \begin{cases}\mathbf{I}_{K} & \text { when } i=l \\ 0 & \text { otherwise }\end{cases}
$$

\subsubsection{Pilot re-use}

In this scenario, we assume that orthogonal pilot sequences are only assigned for the $K$ users in the $i$-th cell, and these sequences are re-used in all the other cells in the system. The pilot sequences used in the $i$-th cell can be represented by a $K \times \tau_{r}$ matrix $\boldsymbol{\Phi}_{i, r}\left(\tau_{r} \geq K\right)$, which satisfies $\boldsymbol{\Phi}_{i, r} \boldsymbol{\Phi}_{i, r}^{\dagger}=\boldsymbol{I}_{K}$. Then, the $M \times \tau_{r}$ received pilot matrix at the $l$-th BS is given by

$$
\boldsymbol{Y}_{l, r}=\sqrt{p_{r}} \sum_{i=1}^{L} \boldsymbol{G}_{l i} \boldsymbol{\Phi}_{i, r}+\boldsymbol{N}_{l, r}
$$

where $p_{r} \triangleq \tau_{r} p_{u}$, and $N_{l, r}$ is the $M \times \tau_{r}$ AWGN matrix at the $l$-th BS with i.i.d $C \mathcal{N}(0,1)$ elements.

After receiving the pilot sequences transmitted by the users in the system, the BSs estimate their own channel matrices by utilising one of the pilot-based channel estimation methods. In

this paper, we assume that minimum mean-square-error (MMSE) channel estimation is used. The MMSE estimate of the channel matrix $\boldsymbol{G}_{l i}$ is given as [11]

$$
\hat{\boldsymbol{G}}_{l i, s}=\sqrt{p_{s}} \boldsymbol{Y}_{l, s}\left(\boldsymbol{I}+\sqrt{p_{s}} \sum_{j=1}^{L} \boldsymbol{\Phi}_{j, s}^{\dagger} \boldsymbol{D}_{l j} \boldsymbol{\Phi}_{j, s}\right)^{-1} \boldsymbol{\Phi}_{i, s}^{\dagger} \boldsymbol{D}_{l i}^{1 / 2},
$$

where $s \in\{r, o\}$.

\subsection{Uplink Data Transmission}

During this phase, the users in the cells transmit their data symbols to their corresponding BSs. Each BS uses its own estimated channel matrix to build the detection matrix. Let $\boldsymbol{A}_{l, s}$ be the $M \times K$ linear detector matrix of the $l$-th BS which is calculated using $\hat{\boldsymbol{G}}_{l l, s}$. In this paper we consider two 
conventional linear detectors, namely, MRC and ZF, giving,

$$
\boldsymbol{A}_{l, s}= \begin{cases}\hat{\boldsymbol{G}}_{l l, s} & \text { for MRC } \\ \hat{\boldsymbol{G}}_{l l, s}\left(\hat{\boldsymbol{G}}_{l l,}^{\dagger} \hat{\boldsymbol{G}}_{l l, s}\right)^{-1} & \text { for ZF }\end{cases}
$$

The $l$-th BS multiply the received signals vector given in (1) with the decoding matrix $\boldsymbol{A}_{l, s}^{\dagger}$ to retrieve the transmitted data symbols as follows

$$
\boldsymbol{z}_{l, s}=\boldsymbol{A}_{l, s}^{\dagger} \boldsymbol{y}_{l}
$$

From (1) and (8), the received signals vector in the $l$-th BS after applying a linear detector can be represented as

$$
\begin{aligned}
\boldsymbol{z}_{l, s} & =\sqrt{p_{u}} \boldsymbol{A}_{l, s}^{\dagger} \sum_{i=1}^{L} \boldsymbol{G}_{l i} \boldsymbol{x}_{i}+\boldsymbol{A}_{l, s}^{\dagger} \boldsymbol{n}_{l} \\
& =\sqrt{p_{u}} \boldsymbol{A}_{l, s}^{\dagger} \boldsymbol{G}_{l l} \boldsymbol{x}_{l}+\sqrt{p_{u}} \boldsymbol{A}_{l, s}^{\dagger} \sum_{\substack{i=1 \\
i \neq l}}^{L} \boldsymbol{G}_{l i} \boldsymbol{x}_{i}+\boldsymbol{A}_{l, s}^{\dagger} \boldsymbol{n}_{l} .
\end{aligned}
$$

\section{Lower Bounds and Spectral Efficiency}

For simplicity, we assume here that all the direct gains are equal to 1 and all the cross gains are equal to $\alpha$, i.e., $\beta_{l l k}=1$ and $\beta_{l i k}=\alpha \forall i \neq l, k=1, \ldots, K$. Owing to the properties of MMSE estimator, the estimation error matrix $\mathcal{E}_{s}$, which is independent of $\hat{\boldsymbol{G}}_{l l, s}$, is given by

$$
\mathcal{E}_{s} \triangleq\left(\boldsymbol{G}_{l l}-\hat{\boldsymbol{G}}_{l l, s}\right)
$$

When $s=o$, then $\boldsymbol{\varepsilon}_{j, s}$ which is the $j$-th column of the matrix $\boldsymbol{E}_{s}$, and $\hat{\boldsymbol{g}}_{l l j, s}$ which is the $j$-th column of the $\hat{\boldsymbol{G}}_{l l, s}$ matrix, are $\mathcal{C N}\left(0, V_{j, o} \boldsymbol{I}_{M}\right)$ and $\mathcal{C N}\left(0,\left(1-V_{j, o}\right) \boldsymbol{I}_{M}\right)$, respectively [5]. While, $\boldsymbol{\varepsilon}_{j, s}$ and $\hat{\boldsymbol{g}}_{l l j, s}$ are $\mathcal{C N}\left(0, V_{j, r} \boldsymbol{I}_{M}\right)$ and $\mathcal{C N}\left(0,\left(1-V_{j, r}\right) \boldsymbol{I}_{M}\right)$ when $s=r$, respectively [11]. Whereas, $V_{j, o}$ and 
$V_{j, r}$ are given as follows

$$
\begin{aligned}
V_{j, o} & =\frac{\beta_{l l j}}{1+p_{o} \beta_{l l j}} \\
& =\frac{1}{1+p_{o}} . \\
V_{j, r}= & \frac{1+p_{r} \sum_{i \neq l}^{L} \beta_{l i j}}{1+p_{r} \sum_{i=1}^{L} \beta_{l i j}} \\
= & \frac{1+p_{r}(L-1) \alpha}{1+p_{r} \bar{L}} .
\end{aligned}
$$

where $\bar{L}=(L-1) \alpha+1$. Clearly, when $L=1$, then (11) is equal to (12).

Substituting (10) in (9), we get

$$
\boldsymbol{z}_{l, s}=\sqrt{p_{u}} \boldsymbol{A}_{l, s}^{\dagger}\left(\hat{\boldsymbol{G}}_{l l, s}+\mathcal{E}_{s}\right) \boldsymbol{x}_{l}+\sqrt{p_{u}} \boldsymbol{A}_{l, s}^{\dagger} \sum_{\substack{i=1 \\ i \neq l}}^{L} \boldsymbol{G}_{l i} \boldsymbol{x}_{i}+\boldsymbol{A}_{l, s}^{\dagger} \boldsymbol{n}_{l} .
$$

Let $z_{l k, s}$ and $x_{i k}$ be the $k$-th elements of the $K \times 1$ vectors $z_{l, s}$ and $\boldsymbol{x}_{i}$, respectively. Then, the signal of the $k$-th user at the $l$-th BS after applying the detector matrix can be expressed as

$$
\begin{aligned}
z_{l k, s}= & \sqrt{p_{u}} \boldsymbol{a}_{l k, s}^{\dagger} \hat{\boldsymbol{s}}_{l k, s} x_{l k} \\
& +\sqrt{p_{u}} \sum_{\substack{j=1 \\
j \neq k}}^{K} \boldsymbol{a}_{l k, s}^{\dagger} \hat{\boldsymbol{g}}_{l l j, s} x_{l j}+\sqrt{p_{u}} \sum_{j=1}^{K} \boldsymbol{a}_{l k, s}^{\dagger} \boldsymbol{\varepsilon}_{j, s} x_{l j} \\
& +\sqrt{p_{u}} \sum_{\substack{i=1 \\
i \neq l}}^{L} \sum_{j=1}^{K} \boldsymbol{a}_{l k, s}^{\dagger} \boldsymbol{g}_{l i j} x_{i j}+\boldsymbol{a}_{l k, s}^{\dagger} \boldsymbol{n}_{l},
\end{aligned}
$$

where $\boldsymbol{a}_{l k, s}$ and $\boldsymbol{g}_{l i k}$ are the $k$-th columns of the matrices $\boldsymbol{A}_{l, s}$ and $\boldsymbol{G}_{l i}$, respectively.

Assuming that the channel is ergodic so that each codeword spans over a large (infinite) number of realizations of the fast-fading factors of the channel matrix $\boldsymbol{G}_{l i}[5]$, then the ergodic achievable 
uplink rates of the $k$-th user in the $l$-th cell, for both scenarios, are given by (15) and (16).

$$
\begin{aligned}
& R_{l k, o}=\mathbb{E}\left\{\log _{2}\left(1+\frac{p_{u}\left|\boldsymbol{a}_{l k, o}^{\dagger} \hat{\boldsymbol{g}}_{l l k, o}\right|^{2}}{p_{u} \sum_{\substack{j=1 \\
j \neq k}}^{K}\left|\boldsymbol{a}_{l k, o}^{\dagger} \hat{\boldsymbol{g}}_{l l j, o}\right|^{2}+p_{u}\left\|\boldsymbol{a}_{l k, o}\right\|^{2} \sum_{j=1}^{K} \frac{\beta_{l l j}}{p_{o} \beta_{l l j}+1}+p_{u} \sum_{\substack{i=1 \\
i \neq l}}^{L} \sum_{j=1}^{K}\left|\boldsymbol{a}_{l k, o}^{\dagger} \boldsymbol{g}_{l i j}\right|^{2}+\left\|\boldsymbol{a}_{l k, o}\right\|^{2}}\right)\right\} . \\
& R_{l k, r}=\mathbb{E}\left\{\log _{2}\left(1+\frac{p_{u}\left|\boldsymbol{a}_{l k, r}^{\dagger} \hat{\boldsymbol{g}}_{l l k, r}\right|^{2}}{p_{u} \sum_{\substack{j=1 \\
j \neq k}}^{K}\left|\boldsymbol{a}_{l k, r}^{\dagger} \hat{\boldsymbol{g}}_{l l j, r}\right|^{2}+p_{u}\left\|\boldsymbol{a}_{l k, r}\right\|^{2} \sum_{j=1}^{K} \frac{1+p_{r} \sum_{i \neq l}^{L} \beta_{l i j}}{1+p_{r} \sum_{i=1}^{L} \beta_{l i j}}+p_{u} \sum_{\substack{i=1 \\
i \neq l}}^{L} \sum_{j=1}^{K}\left|\boldsymbol{a}_{l k, r}^{\dagger} \boldsymbol{g}_{l i j}\right|^{2}+\left\|\boldsymbol{a}_{l k, r}\right\|^{2}}\right)\right\} .
\end{aligned}
$$

\subsection{Lower Bounds on Achievable Rates}

\subsubsection{Orthogonal Pilot}

- For MRC with Rayleigh fading, and $M \geq 2$, the uplink achievable rate of the $k$-th user in the $l$-th cell is lower bounded by

$$
\tilde{R}_{l k, o}^{M R C}=\log _{2}\left(1+\frac{p_{u}\left(1-V_{k, o}\right)(M-1)}{p_{u}\left(1-V_{k, o}\right)(K-1)+p_{u} K V_{k, o}+p_{u}(L-1) K \alpha+1}\right) .
$$

Proof: See Appendix A.

- For ZF with Rayleigh fading, and $M \geq K+1$, the lower bound on the uplink achievable rate of the $k$-th user in the $l$-th cell is given by

$$
\tilde{R}_{l k, o}^{Z F}=\log _{2}\left(1+\frac{p_{u}\left(1-V_{k, o}\right)(M-K)}{p_{u} K V_{k, o}+p_{u}(L-1) K \alpha+1}\right) .
$$

Proof: See Appendix B. 


\subsubsection{Pilot Re-use}

For the pilot re-use scenario, we will use the lower bounds given in [5], which are given by (19) and (20) for MRC and ZF, respectively.

$$
\begin{gathered}
\tilde{R}_{l k, r}^{M R C}=\log _{2}\left(1+\frac{p_{u}^{2}(M-1) \tau_{r}}{\tau_{r}\left(K \bar{L}^{2}-1+\alpha(\bar{L}-1)(M-2)\right) p_{u}^{2}+\bar{L}\left(K+\tau_{r}\right) p_{u}+1}\right) \\
\tilde{R}_{l k, r}^{Z F}=\log _{2}\left(1+\frac{p_{u}^{2}(M-K) \tau_{r}}{\tau_{r} K\left(\bar{L}^{2}-\alpha \bar{L}+\alpha-1\right) p_{u}^{2}+\tau_{r} \alpha^{2}(L-1)(M-K) p_{u}^{2}+\bar{L}\left(K+\tau_{r}\right) p_{u}+1}\right) .
\end{gathered}
$$

\subsection{Spectral Efficiency}

We define the spectral efficiency of the $l$-th cell as the sum of rates achieved by all the users of the $l$-th cell measured in bits/channel use. Thus the spectral efficiency of the $l$-th cell can be expressed as

$$
S E_{l, s}^{A}=\frac{T-\tau_{s}}{T} \sum_{k=1}^{K} \tilde{R}_{l k, s}^{A},
$$

where $A \in\{M R C, Z F\}$ corresponds to $\mathrm{MRC}$ and $\mathrm{ZF}$, and $T$ is the coherence interval of the channel measured in symbols. In (21), one can predict that there is a trade-off between the length of the pilot sequence assigned for each user and the spectral efficiency. In Section 6 we discuss this trade-off in more details.

\section{Pilot Allocation Algorithm}

In this section, we propose an algorithm for pilot sequences allocation to mitigate the effect of pilot contamination. For simplicity and without loss of generality, we assume that all cells in the system have the same number of users $K$ and that $\tau>K$, which is the case when either the number of users is not very large, or when the system allocates more resources for the channel estimation process 
in order to reduce the effect of pilot contamination, as suggested in the first scenario. Moreover, we assume that all BSs are connected to a centralized control unit, and that all the direct gains are made available to this unit as it controls the pilot sequence allocation for the different users in the different cells.

Denoting by $\boldsymbol{q} \in \mathbb{R}^{K L \times 1}$ the vector of the direct gain values of all the users in the system sorted in an ascending order (here we assume that the different direct gains have different values), then in the first step of the algorithm $\tau-K$ out of $\tau$ pilot sequences are assigned to the first $\tau-K$ users associated with the least direct gain values in vector $\boldsymbol{q}$, while the remaining $K$ pilot sequences are preserved. Once a user is assigned with a pilot sequence, then both the user and the assigned pilot sequence are removed from the users and the pilot sequences pool, respectively. Moreover, the algorithm saves the index (ix) of the last direct gain value in vector $\boldsymbol{q}$ associated with the user that has been assigned with a pilot sequence. In the second step, the algorithm checks the number of users that have not been assigned with a pilot sequence in each cell. If the maximum number (max) is less than $K$, then the algorithm assigns $K-\max$ pilot sequences out of the $K$ preserved sequences to $K-\max$ users with the least direct gain values in vector $\boldsymbol{q}$ starting from the index $(i x)$. The second step is repeated until the number of the preserved pilot sequences becomes equal to the maximum number of users among all cells $(\max )$. Then in the third step, the algorithm assigns the remaining pilot sequences to the remaining users of all cells. In other words, the remaining set of users in all cells are sharing the remaining set of pilot sequences. We emphasize here that at the end of the algorithm, each user in the system is assigned only one pilot sequence, which is orthogonal to the sequences assigned to the other users in the same cell.

The reasons behind choosing the users with the least direct gains are: firstly to improve the achievable rate of these users as they have the least channel gains. Secondly, these users are usually located at the edge of the cells, thus they tend to have large cross gains, which means they cause higher inter-cell interference. Therefore, by allocating orthogonal pilot sequences to the users of this type we improve their achievable rates and reduce the inter-cell interference caused to the users in other cells. The proposed algorithm requires the direct gains values solely, which change 
slowly over time and can be obtained and tracked easily with low complexity [2]. Moreover, the pilot allocation process should be done by a centralized control unit. These requirements are already met in the recent wireless communication standards, which makes the implementation of this algorithm very amenable [12].

\section{Numerical Results}

\subsection{Scenario I}

In this scenario, we consider a system with $L=7$ cells that share the same time-frequency resources. The centre cell, surrounded by other cells, is considered as the target cell. We assume that the lengths of the pilot sequences used in the pilot re-use and orthogonal pilot scenarios are $\tau_{r}=K$ and $\tau_{o}=L K$, respectively. We further assume that the transmit SNR of each user $p_{u}=10 \mathrm{~dB}$, and the coherence interval of the channel $T=196$ unless stated otherwise.

Fig. 1 shows the proposed bounds and the simulated spectral efficiencies versus the number of BS antennas for both scenarios and for both MRC and ZF, with $\alpha=0.5$ and $K=20$. It is clear from this figure that all the bounds are very tight. We can also notice in Fig. 1 the significant impact of pilot contamination on the performance of the system. The spectral efficiency starts to saturate as we increase the number of BS antennas for the pilot re-use scenario. On the other hand, it increases as we increase the number of BS antennas for the orthogonal pilot scenario. This is due to the fact that for the pilot re-use scenario there is a residual interference coming from the users in the other cells that are re-using the same pilot sequences, and this interference can not be eliminated by simply increasing the number of BS antennas.

To investigate the effect of number of users on the system performance, we show the spectral efficiency versus the number of users $K$ in Fig. 2, for $\alpha=0.5$ and $M=100$. We can see that for a small number of users, the use of orthogonal pilot sequences in the different cells yields better spectral efficiency than re-using the same pilot sequences in all cells. In other words, allocating more resources for the training phase to avoid the problem of pilot contamination is more feasible 


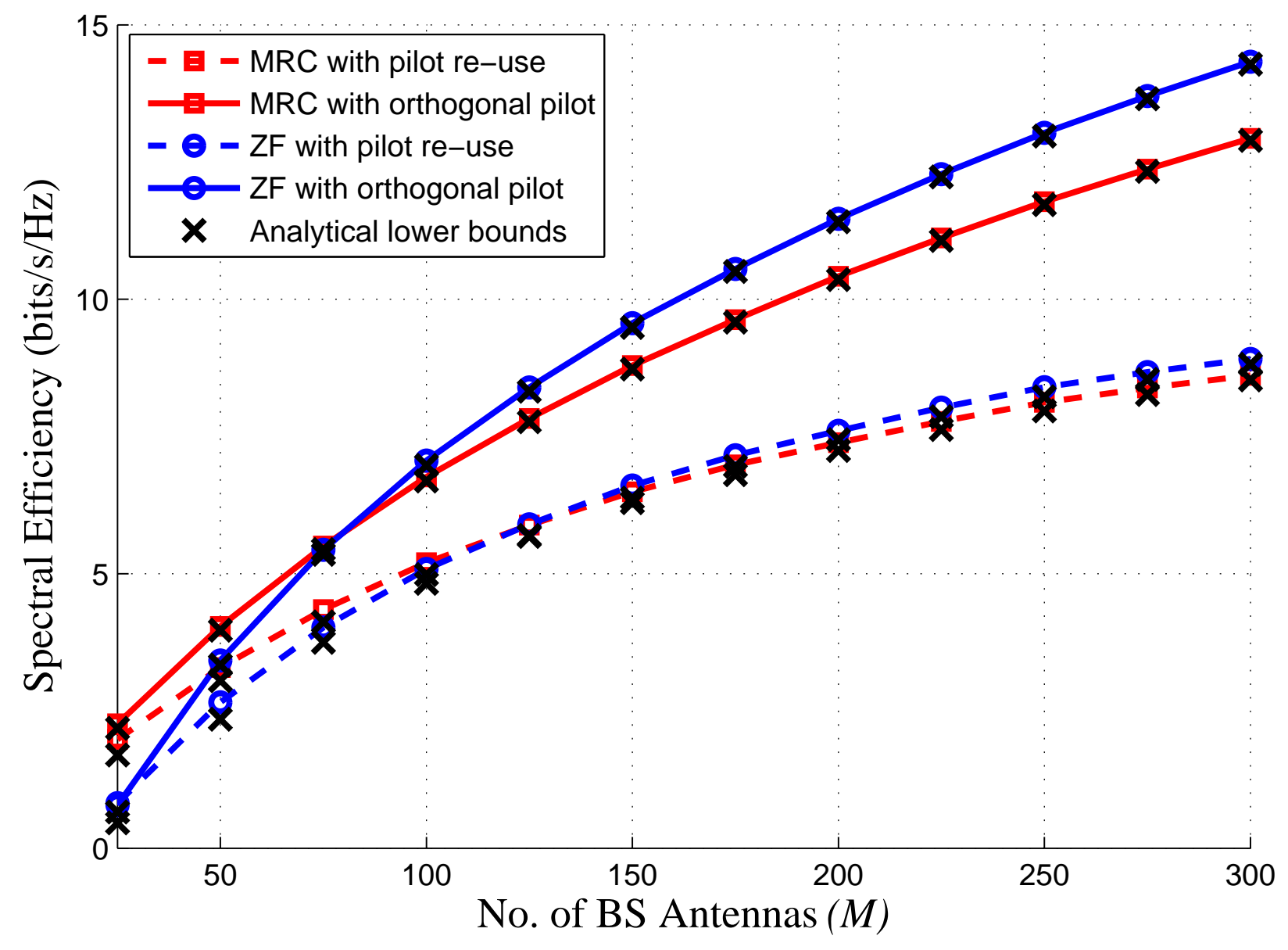

Fig. 1. Spectral efficiency versus the number of BS antennas $M$.

than using these resources for data transmission. However, as the number of users increases, the spectral efficiency decreases for the orthogonal pilot scenario and for both the MRC and ZF detectors. For large number of users (around 23 users), the spectral efficiency achieved with the pilot re-use scenario becomes larger than that achieved with orthogonal pilot scenario, which indicates that it is more beneficial to assign more resources for the data transmission rather than for training.

We next examine the effect of pilot contamination. Fig. 3 shows the spectral efficiency versus the cross gain $\alpha$, for $M=50$ and $K=20$. We can see that as the cross gain value increases, the system performance degrades significantly for the pilot re-use scenario, especially when the cross gain value becomes close to the direct gain value. This is due to the fact that as the cross gain value increases, the contamination in the channel estimation increases too, which results in a higher inter-cell interference. On the other hand, the effect of increasing the cross gain value is 


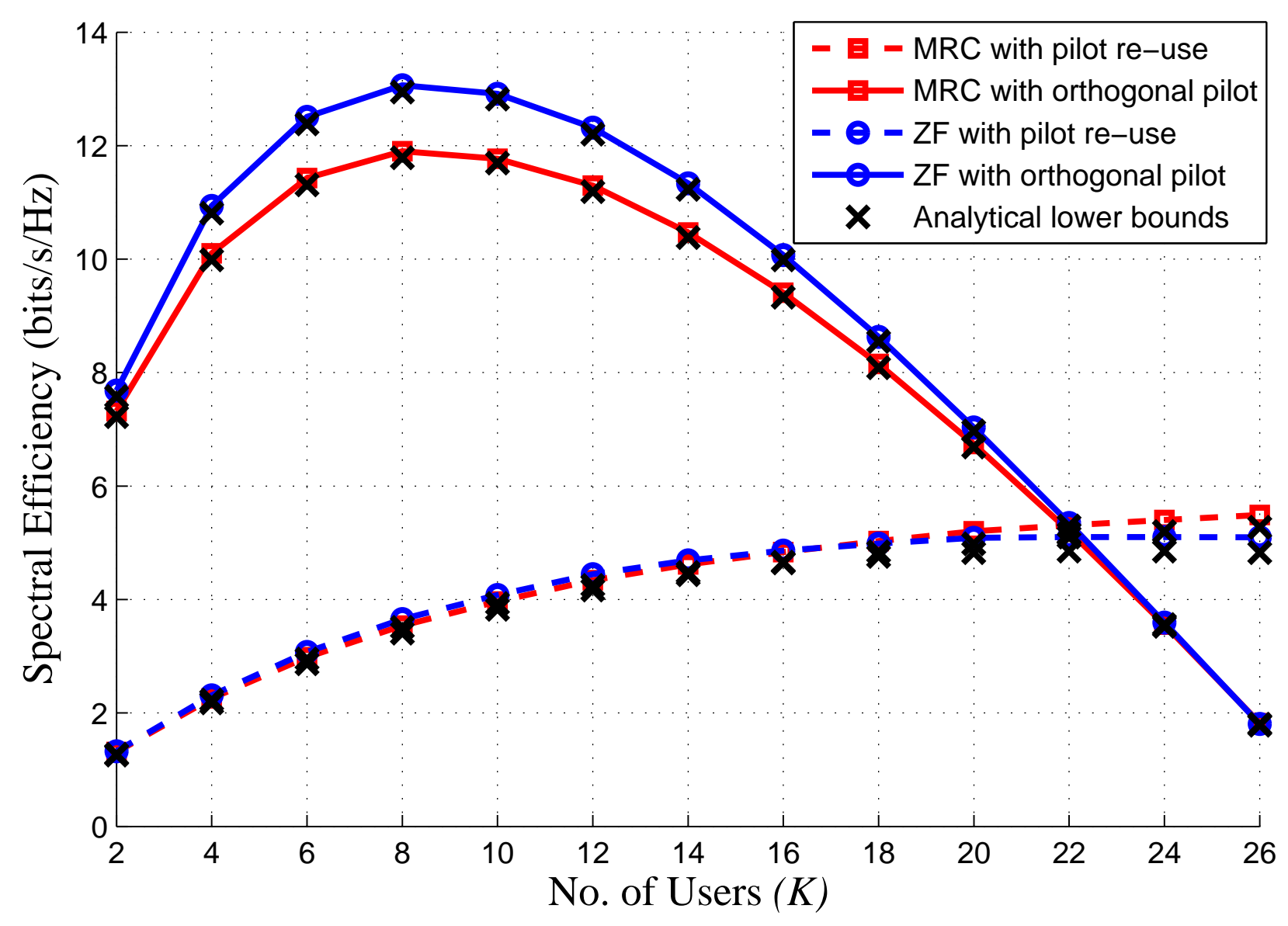

Fig. 2. Lower bound on the spectral efficiency versus the number of users $K$.

less significant on the system's performance for the orthogonal pilot scenario as there is no pilot contamination in this case. However, there is still inter-cell interference coming from the users in the other cells which increases with the cross gain value. Furthermore, we can notice that when the cross gain value is small compared to the direct gain value, the system's performance with the pilot re-use scenario is better than its performance with the orthogonal pilot scenario. However, as the cross gain value increases, the opposite holds true. This indicates that when the inter-cell interference resulting from pilot contamination is high, then it is more beneficial to utilize more system's resources to eliminate this interference (by using longer pilot sequences) rather than using these resources for transmitting data. The fact that MRC is preferable over ZF in low SINR is illustrated in Fig. 3. Whereas, for high cross gain values, the SINR decreases due to the inter-cell interference. Thus, the system performance with ZF degrades dramatically. 


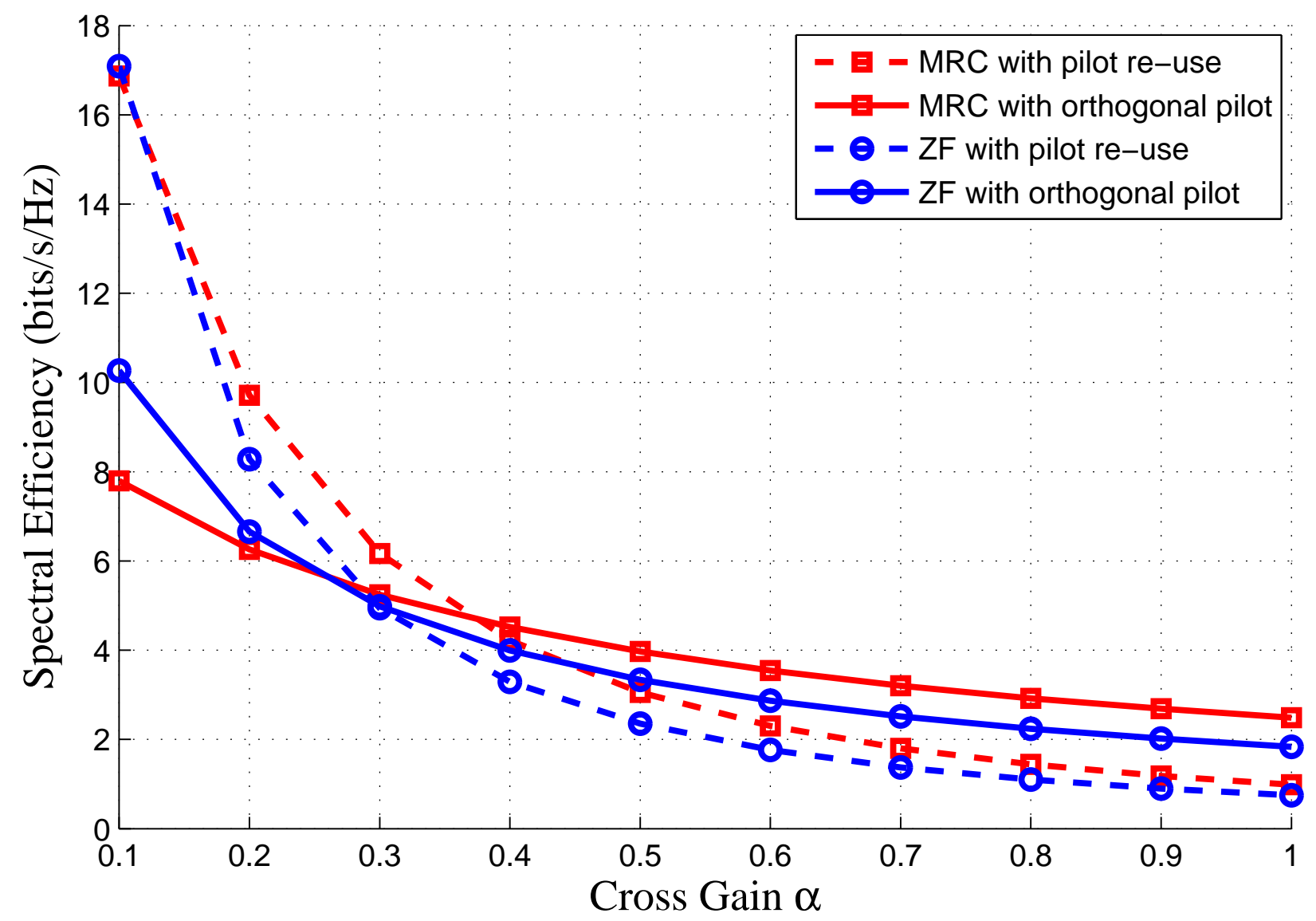

Fig. 3. Spectral efficiency versus the cross gain.

Finally, we show the spectral efficiency versus the length of the coherence interval $T$, for $\alpha=$ $0.5, M=160$, and $K=20$. In Fig. 4, it is noticeable that for short coherence intervals (high mobility environment), it is better to re-use the same pilot sequences in the different cells as this will reduce the length of the pilot sequence allocated for each user, which means sustaining more resources for data transmission. However, for moderate and large coherence intervals, the use of orthogonal pilot sequences becomes more feasible as it results in a better performance compared to the pilot re-use case.

\subsection{Scenario II}

In this scenario, we investigate the performance of the algorithm proposed in Section 5. We consider a cellular network with $L=3$ circle cells. We assume that the radius of each cell is 


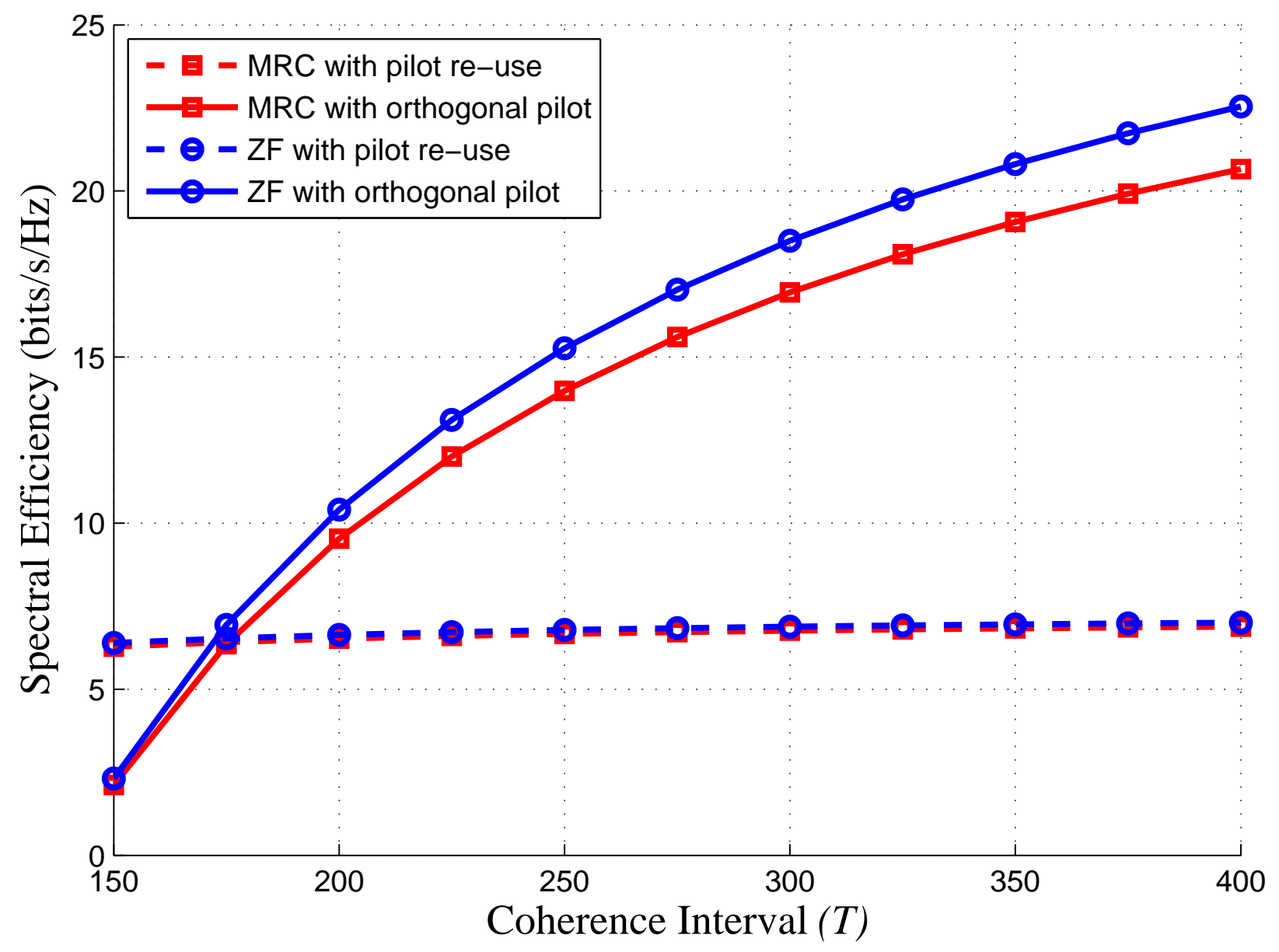

Fig. 4. Spectral efficiency versus the coherence interval.

$\operatorname{Rad}_{B S}=500$ metres. We further assume that the users of each cell are uniformly distributed around the BS of that cell. As addressed in [13], the large-scale fading coefficient between the $k$-th user in the $i$-th cell and the $l$-th BS can be modelled as

$$
\beta_{l i k}=\frac{\gamma_{k}}{\left(d_{i k} / \operatorname{Rad}_{B S}\right)^{v}}
$$

where $\gamma_{k}$ represents the shadowing which is a log-normal random variable with standard deviation $\sigma=8 \mathrm{~dB}, d_{l i k}$ is the distance between the $k$-th user in the $i$-th cell and the $l$-th BS, and $v=3$ is the path loss exponent. For simplicity, we assume that $T=200$, and we only consider the ZF detector.

Fig. 5 plots the cumulative distribution function (CDF) curves of both the minimum and the mean uplink achievable rates with $M=200, K=10$, and $\tau=0.1 T=20$. Obviously, we can see 


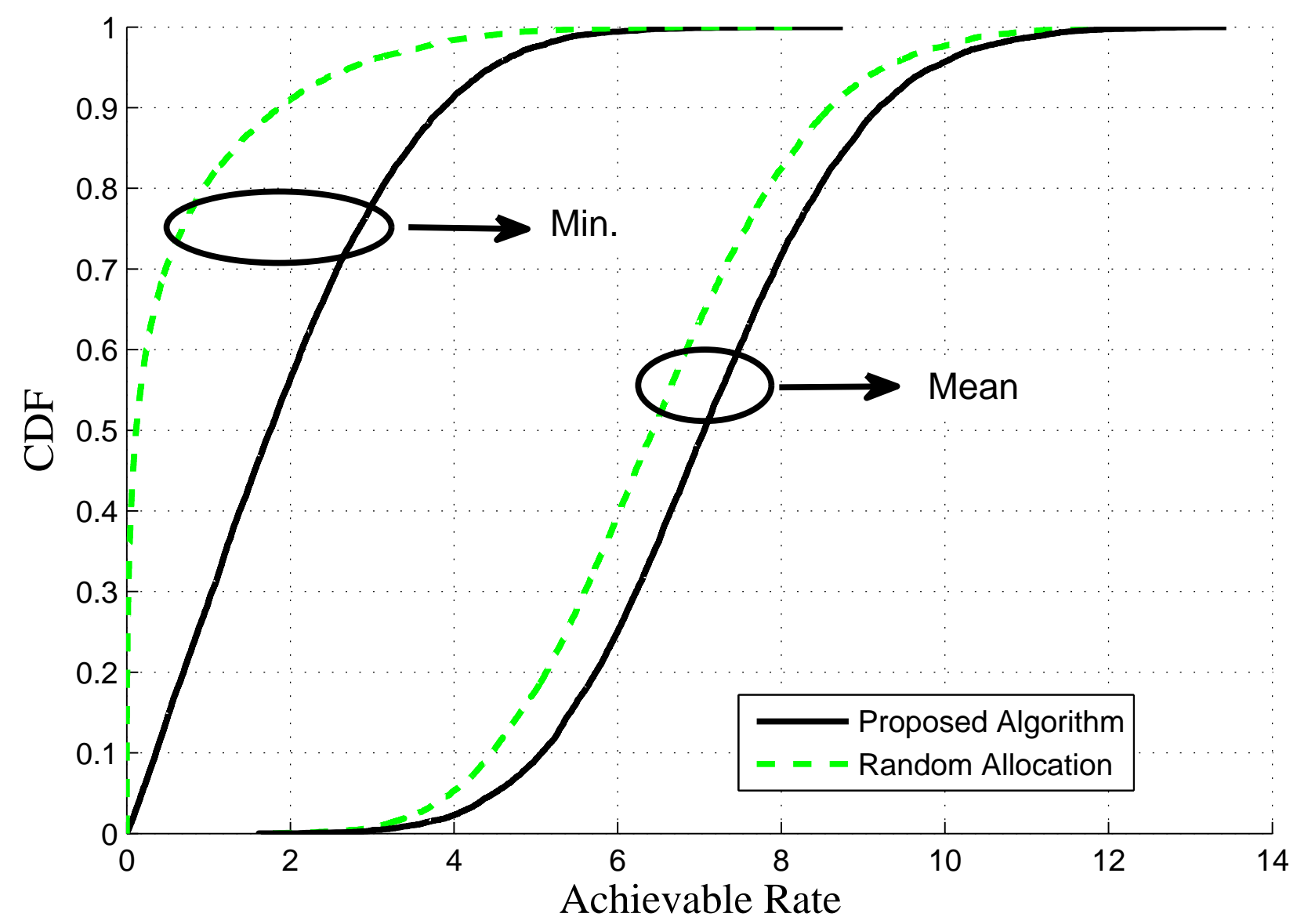

Fig. 5. A comparison between the CDF of the minimum and mean uplink achievable rates of the proposed algorithm versus random pilot allocation.

that the proposed algorithm significantly improves the minimum achievable rate compared with the conventional random allocation method which refers to the case when the pilot sequences are allocated to the users with neither prioritization nor favouritism for a specific user or group of users over the others during the pilot sequences allocation process [2]. We can also note that the propsed algorithm improves the mean achievable rate. This is due to the fact that the proposed algorithm assigns orthogonal pilot sequences to the users with the smallest direct gains, which in turn increases their SINRs and improves their achievable rates. Also by ensuring that these assigned orthogonal sequences are not re-used by any other user, the algorithm reduces the intercell interference which improves the total achievable rates.

In Fig. 6, we investigate the effect of increasing the number of BS antennas on the performance of the system with the use of the proposed algorithm, for $K=15$ and $\tau=0.1 T=20$. It is clearly 


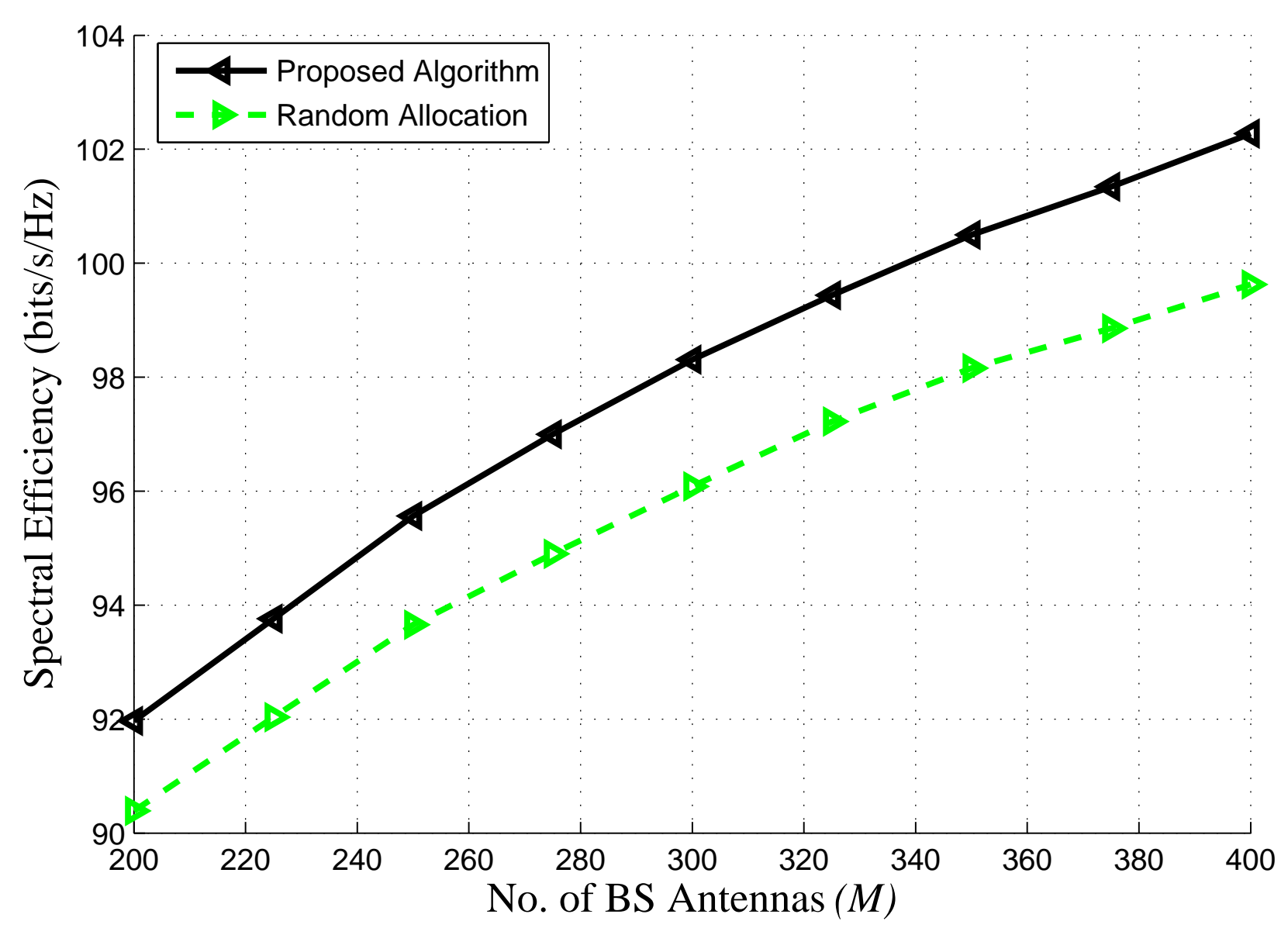

Fig. 6. The effect of increasing the number of BS antennas on the spectral efficiency.

shown that as the the number of BS antennas increases, the performance of the system improves for both the conventional (random) pilot allocation and the proposed algorithm. However, as the number of BS antennas becomes large, the gap between the performance of random allocation and the proposed algorithm becomes larger. Whereas for $M=200$, the gap is about $1.5 \mathrm{bits} / \mathrm{s} / \mathrm{Hz}$. While for $M=400$, the gap is about $3 \mathrm{bits} / \mathrm{s} / \mathrm{Hz}$. This is because the effect of pilot contamination cannot be eliminated by increasing the number of BS antennas for the case of random pilot allocation. Thus the system performance starts to saturate as the number of BS antennas increases. While with the use of the proposed algorithm, the effect of pilot contamination is alleviated. 


\section{Conclusions}

In this paper, we have considered two different scenarios for pilot signals allocation in TDD multicell massive MIMO systems and compared between them in terms of spectral efficiency. We have also derived lower bounds on the achievable rate for MRC and ZF detectors. Our results have shown that increasing the number of BS antennas alone can not resolve the problem of pilot contamination, and that when the effect of pilot contamination is very significant, the use orthogonal pilot sequences would results in a better system performance. Furthermore, we have shown that when the cross gain value is high and the number of BS antennas is not much greater than the number of users, then MRC performs better than ZF. Moreover, when the coherence time of the channel is large (limited mobility environment), then the use of orthogonal pilot sequences yields better system performance compared to the pilot re-use case. Also, we have demonstrated that the proposed pilot sequence allocation scheme improves the system performance compared to conventional scheme.

\section{References}

1 Gesbert, D., Kountouris, M., Heath, R. and et al.: 'Shifting the MIMO Paradigm', IEEE Sig. Process. Mag., 2007, 24, (5), pp. 36-46.

2 Marzetta, T.: 'Noncooperative Cellular Wireless with Unlimited Numbers of Base Station Antennas', IEEE Trans. Wireless Commun., 2010, 9, (11), pp. 3590-3600.

3 Larsson, E., Edfors, O., Tufvesson, F. and et al.: 'Massive MIMO for next generation wireless systems', IEEE Commun. Mag., 2014, 52, (2), pp. 186-195.

4 Hoydis, J., Hoek, C., Wild, T. and et al.: 'Channel measurements for large antenna arrays'. Proc. Proc. IEEE Int. Sympos. Wireless Commun. Syst. (ISWCS), 2012, pp. 811-815.

5 Ngo, H. Q., Larsson, E. and Marzetta, T.: 'Energy and Spectral Efficiency of Very Large Multiuser MIMO Systems', IEEE Trans. Commun., 2013, 61, (4). 
6 Lu, L., Li, G., Swindlehurst, A. and et al.: 'An Overview of Massive MIMO: Benefits and Challenges', IEEE J. Sel. Topics Sig. Process., 2014, 8, (5), pp. 742-758.

7 Huh, H., Moon, S. H., Kim, Y. T. and et al.: 'Multi-Cell MIMO Downlink With Cell Cooperation and Fair Scheduling: A Large-System Limit Analysis', IEEE Trans. on Info. Theory, 2011, 57, (12), pp. 7771-7786.

8 Yin, H., Gesbert, D., Filippou, M. and et al.: 'A Coordinated Approach to Channel Estimation in Large-Scale Multiple-Antenna Systems', IEEE J. Sel. Areas Commun., 2013, 31, pp. 264273.

9 Li, M., Jin, S. and Gao, X.: 'Spatial orthogonality-based pilot reuse for multi-cell massive MIMO transmission'. Proc. Int. Conf. on Wireless Commun. Sig. Process. (WCSP), 2013, pp. 1-6.

10 Zhu, X., Wang, Z., Qian, C., Dai, L., Chen, J., Chen, S. and Hanzo, L.: 'Soft Pilot Reuse and Multicell Block Diagonalization Precoding for Massive MIMO Systems', IEEE Trans. Veh. Techno, 2016, 65, (5), pp. 3285-3298.

11 Jose, J., Ashikhmin, A, Marzetta, T. and et al.: 'Pilot Contamination and Precoding in MultiCell TDD Systems', IEEE Trans. Wireless Commun., 2011, 10, (8), pp. 2640-2651.

12 Sesia, S., Toufik, I. and Baker, M.: LTE-The UMTS Long Term Evolution: From Theory to Practice (Wiley., Chichester, 2009, 1st edn.).

13 Rusek, F., Persson, D., Lau, B. K. and et al.: 'Scaling Up MIMO: Opportunities and Challenges with Very Large Arrays', IEEE Sig. Process. Mag., 2013, 30, (1), pp. 40-60.

14 Tulino, A. M. and Verdu, S.: Random matrix theory and wireless communications (Now Publishers Inc., New York, 2004, 1st edn.). 


\section{Appendix A: MRC lower bound}

By the convexity of $\log _{2}\left(1+\frac{1}{x}\right)$ and using Jensen's inequality, and by substituting $\boldsymbol{a}_{l k, o}=\hat{\boldsymbol{g}}_{l l k, o}$ in (15), we get (23).

We can write the expectation in (23) as (24). Then, by conditioning on $\hat{\boldsymbol{g}}_{l l k, o}$, we can get:

- The $1^{\text {st }}$ term and the $2^{\text {nd }}$ term have an exponential distribution [14], thus the $\mathbb{E}\left\{1^{\text {st }}\right.$ term $\}=$ $\left(1-V_{k, o}\right)$, while the $\mathbb{E}\left\{2^{\text {nd }}\right.$ term $\}=\beta_{l i j}$.

- The $3^{r d}$ term has an inverse chi-square distribution [14], with $\mathbb{E}\left\{3^{r d}\right.$ term $\}=\frac{1}{p_{u}\left(1-V_{k, o}\right)(M-1)}$.

Therefore, substituting these values into (24) and (23), we can get the desired equation given in (17).

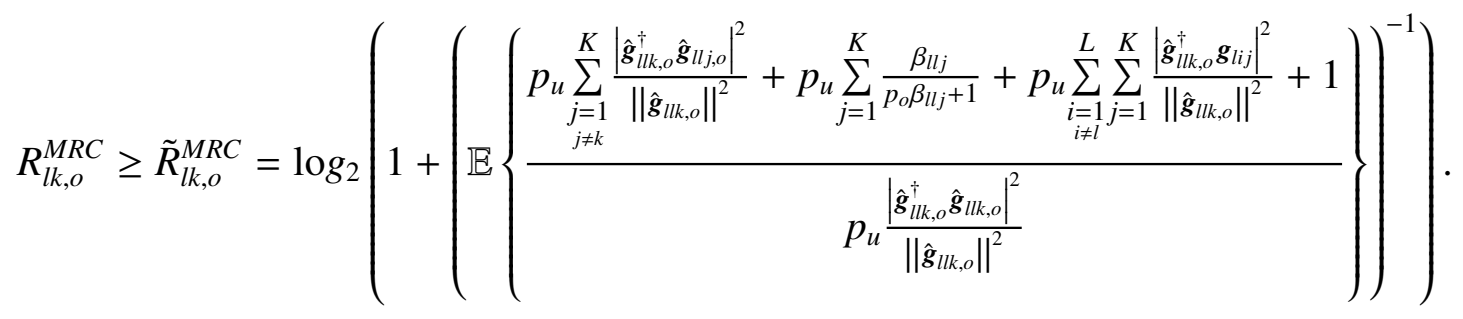

$$
\begin{aligned}
& \mathbb{E}\left\{\frac{p_{u} \sum_{\substack{j=1 \\
j \neq k}}^{K} \frac{\left|\hat{\boldsymbol{g}}_{l l k, o}^{\dagger} \hat{\boldsymbol{s}}_{l l, o}\right|^{2}}{\left\|\hat{\boldsymbol{g}}_{l l k, o}\right\|^{2}}+p_{u} \sum_{j=1}^{K} \frac{\beta_{l l j}}{p_{o} \beta_{l l j}+1}+p_{u} \sum_{\substack{i=1 \\
i \neq l}}^{L} \sum_{j=1}^{K} \frac{\left|\hat{\boldsymbol{g}}_{l k, o}^{\dagger} \boldsymbol{g}_{l i j}\right|^{2}}{\left\|\hat{\boldsymbol{g}}_{l l k, o}\right\|^{2}}+1}{p_{u} \frac{\left|\hat{\boldsymbol{g}}_{l k, o}^{\dagger} \hat{\boldsymbol{g}}_{l l k, o}\right|^{2}}{\left\|\hat{\boldsymbol{g}}_{l l k, o}\right\|^{2}}}\right\}
\end{aligned}
$$

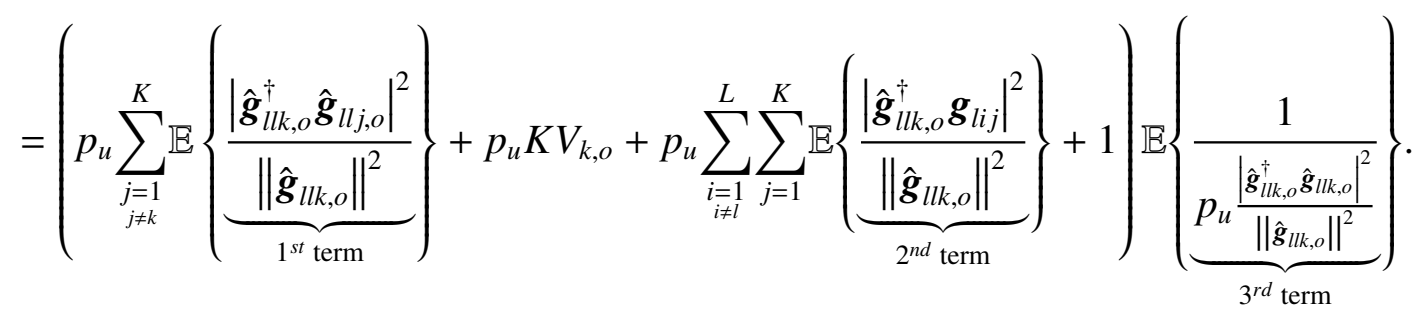




\section{Appendix B: ZF lower bound}

By substituting $\boldsymbol{a}_{l k, o}=\hat{\boldsymbol{g}}_{l l k, o}\left(\hat{\boldsymbol{g}}_{l l k, o}^{\dagger} \hat{\boldsymbol{g}}_{l l k, o}\right)^{-1}$ in (15), we get (27). Then, by using Jensen's inequality, we can write the lower bound as (28). The expectation in (28) can be written as (29). Similarly as in Appendix A, by condition on $\boldsymbol{a}_{l k, o}$ we can find

$$
\mathbb{E}\left\{\frac{\left|\boldsymbol{a}_{l k, o}^{\dagger} \boldsymbol{g}_{l i j}\right|^{2}}{\left\|\boldsymbol{a}_{l k, o}\right\|^{2}}\right\}=\beta_{l i j} .
$$

It is known that $\left\|\boldsymbol{a}_{l k, o}\right\|^{2}=\left[\left(\boldsymbol{G}^{H} \boldsymbol{G}\right)^{-1}\right]_{k k}$. Therefore,

$$
\begin{aligned}
\mathbb{E}\left\{\left[\left(\boldsymbol{G}^{H} \boldsymbol{G}\right)^{-1}\right]_{k k}\right\} & =\mathbb{E}\left\{\frac{\left[\left(\boldsymbol{H}^{H} \boldsymbol{H}\right)^{-1}\right]_{k k}}{\left(1-V_{k, o}\right)}\right\}, \\
& =\mathbb{E}\left\{\frac{\operatorname{tr}\left[\left(\boldsymbol{H}^{H} \boldsymbol{H}\right)^{-1}\right]}{K\left(1-V_{k, o}\right)}\right\}, \\
& =\frac{1}{(M-K)\left(1-V_{k, o}\right)}
\end{aligned}
$$

Finally, substituting (25) and (26) into (29) and (28) we can find (18).

$$
\begin{aligned}
& R_{l k, o}^{Z F} \geq \tilde{R}_{l k, o}^{Z F}=\mathbb{E}\left\{\log _{2}\left(1+\frac{p_{u}}{p_{u}\left\|\boldsymbol{a}_{l k, o}\right\|^{2} \sum_{j=1}^{K} \frac{\beta_{l l j}}{p_{o} \beta_{l j}+1}+p_{u} \sum_{\substack{i=1 \\
i \neq l}}^{L} \sum_{j=1}^{K}\left|\boldsymbol{a}_{l k, o}^{\dagger} \boldsymbol{g}_{l i j}\right|^{2}+\left\|\boldsymbol{a}_{l k, o}\right\|^{2}}\right)\right\} . \\
& \tilde{R}_{l k, o}^{Z F}=\log _{2}\left(1+\mathbb{E}\left\{\frac{\frac{p_{u}}{\left\|\boldsymbol{a}_{l k, o}\right\|^{2}}}{p_{u} \sum_{j=1}^{K} \frac{\beta_{l l j}}{p_{o} \beta_{l l j}+1}+p_{u} \sum_{\substack{i=1 \\
i \neq l}}^{L} \sum_{j=1}^{K} \frac{\left|\boldsymbol{a}_{l k, o}^{\dagger} \boldsymbol{g}_{l i j}\right|^{2}}{\left\|\boldsymbol{a}_{l k, o}\right\|^{2}}+1}\right\}\right) .
\end{aligned}
$$




$$
\mathbb{E}\left\{\frac{\frac{p_{u}}{\left\|\mathbf{a}_{l k, o}\right\|^{2}}}{p_{u} K V_{k, o}+p_{u} \sum_{\substack{i=1 \\ i \neq l}}^{L} \sum_{j=1}^{K} \frac{\left|\boldsymbol{a}_{l k, o}^{\dagger} \boldsymbol{g}_{l i j}\right|^{2}}{\left\|\boldsymbol{a}_{l k, o}\right\|^{2}}+1}\right\}=\mathbb{E}\left\{\frac{p_{u}}{\left\|\boldsymbol{a}_{l k, o}\right\|^{2}}\right\}\left(\frac{1}{p_{u} K V_{k, o}+p_{u} \sum_{\substack{i=1 \\ i \neq l}}^{L} \sum_{j=1}^{K} \mathbb{E}\left\{\frac{\left|\boldsymbol{a}_{l k, o}^{\dagger} \boldsymbol{g}_{l i j}\right|^{2}}{\left\|\boldsymbol{a}_{l k, o}\right\|^{2}}\right\}+1}\right) .
$$

